\title{
21. LIGHT OF THE NIGHT SKY / LUMIERE DU CIEL NOCTURNE
}

President: M. S. Hanner

Vice President: M. G. Hauser

Organizing Committee: S. Bowyer, R. Dumont, Yu. Galperin, S. S. Hong, Ph. Lamy, Ch. Leinert, A. C. Levasseur-Regourd, T. Mukai

\section{INTRODUCTION}

The diffuse components of the light of the night sky encompass a variety of physical phenomena over the full range of cosmic distance scales. From the Earth outward they include airglow in the Earth's atmosphere, scattering and thermal emission from the interplanetary dust cloud (zodiacal light), integrated starlight, diffuse galactic light, and extragalactic background radiation. These components are necessarily observed together along the line of sight. Thus, the successful measurement of any one component requires knowledge of all the diffuse components. Moreover, the brightness of the diffuse sky background limits the detection of very faint objects, both extended and point-like. Thus, the spectral and spatial characteristics of the diffuse components have to be known in order to maximize detectability of very faint objects. Commission 21 is the only organization in which all of the diffuse components are studied and compared.

The purview of Commission 21 covers the ultraviolet, visible, and infrared spectral regions. The main sources are the same and the observing techniques are generally similar in these spectral regions. Knowledge gained in one spectral region applies to the others. For example, he spatial distribution of interplanetary dust derived from visible light observations is applicable to analyzing the thermal emission from the dust.

Commission 21 periodically sponsors meetings related to the diffuse astronomical radiations. IAU Symposium 139 "The Galactic and Extragalactic Background Radiation" was held in Heidelberg in 1989 (GEBR) and IAU Colloquium 126 "Origin and Evolution of Interplanetary Dust" took place in Kyoto in 1990 (OED). A Symposium on "Examining the Big Bang and the Diffuse Background Radiations" will be held in conjunction with the IAU General Assembly in 1994. IAU Colloquium 154 "Physics, Chemistry and Dynamics of the Interplanetary Dust" is planned for 1995.

The following abbreviations are used in citing references.

$\begin{array}{lll}\text { A\&A } & \text { Astronomy \& Astrophysics } & \text { GRL } \\ \text { Adv Sp Res } & \text { Advances in Space Research } & \text { JGR Jour. Geophys. Research } \\ \text { AJ } & \text { Astronomical Journal } & \text { JGRP Jour. Geophys. Research, Planets } \\ \text { ApJ } & \text { Astrophysical Journal } & \\ \text { GEBR } & \text { IAU Symposium 139, S. Bowyer and C. Leinert eds., Kluwer, Dordrecht, } \\ & \text { 1990 } \\ \text { OED } & \text { IAU Colloq. 126, A. C. Levasseur-Regourd ed. Kluwer, Tokyo, 1991 } \\ \text { MPB } & \text { Meteoroids and their Parent Bodies, J. Stohl and I. P. Williams eds., Slovak } \\ & \text { Acad. Sci., Bratislava, 1993 }\end{array}$




\section{AIRGLOW}

Research on aeronomical topics related to atmospheric airglow are primarily in the domain of geophysics. However, the brightness and fluctuations in the airglow are important for low-light level astronomical observations, both from the Earth's surface and from low Earth orbit. The Berkeley group have shown that airglow forms a very significant background for diffuse imaging at 1400 to $1800 \AA$ from the shuttle altitude (Lampton et al., GRL 20, 539, 1993).

Ultraviolet spectra of the tropical oxygen nightglow in the range of 830 to $1850 \AA$ at $3 \AA$ resolution were obtained with the Hopkins Ultraviolet Telescope during the Astro-1 shuttle mission in December 1990 (Feldman et al., GRL 19, 453, 1992). The dominant features of the spectrum, other than geocoronal hydrogen, are $O I$ at 1304 and $1356 \AA$ and the radiative recombination continuum near $911 \AA$. The continuum is resolved and found to be consistent with an electron temperature of 1000 to $1250 \mathrm{~K}$. Weak O I emission at $989 \AA$ is also detected, but there is no evidence for any similarly produced atomic nitrogen emissions. Upper limits on shuttle-induced ultraviolet emissions were published by Morrison, Feldman, and Henry (JGR 97, 1633, 1992).

\section{ZODIACAL LIGHT}

The zodiacal light is due to sunlight scattered by interplanetary dust particles. Thermal emission from the dust particles gives rise to the infrared zodiacal emission. The IAU Colloquium 126 "Origin and Evolution of Interplanetary Dust", August 1990, was covered in the previous triennial report.

Search for dust rings around the Sun during the 1991 solar eclipse

The past 25 years have witnessed many attempts to detect thermal emission from a possible dust ring around the Sun with contradictory results. Until the 1991 eclipse, all prior infrared experiments used single-element detectors to scan the corona along the ecliptic to look for excess surface brightness peaks. The availability of relatively large format infrared array detectors now provides the clear advantage of mapping the brightness and polarization of the corona. The 11 July 1991 eclipse path included the high altitude Mauna Kea Observatory, a further advantage to detect the corona at large distances. Two experiments were performed with $\mathrm{HgCdTe}$ array detectors through the broadband $\mathrm{J}, \mathrm{H}$ and $\mathrm{K}$ filters (1.2 to $2.2 \mu \mathrm{m}$ ) (Lamy et al. Science 257, 1377, 1992; Hodapp, MacQueen, and Hall, Nature 355, 707, 1992). Although the sky conditions were not ideal, the $\mathrm{H}$ and $\mathrm{K}$ surface brightnesses clearly show the inhomogeneous structure in the $\mathrm{K}$ corona and the flattening of the $\mathrm{F}$-corona toward the ecliptic but no evidence of a circumsolar, local dust component out to 15 solar radii. Thus, previous observations of thermal emission peaks may have been due to transitory phenomena, such as the breakup of sun-grazing comets. The eclipse data indicate a temperature of near-solar dust slightly below the blackbody temperature and a wide extended zone of sublimation from about 10 solar radii inward even to 3 solar radii (Mann \& MacQueen A \& A, in press, 1993).

\section{Doppler shifts in the zodiacal light}

Mukai and Mann (A\&A 271, 530, 1993) have answered a puzzle of many years standing regarding the observed Doppler shifts in zodiacal light spectra: why dust particles within $0.7 \mathrm{AU}$ 
of the Sun scatter sunlight as if they have orbital velocities greater than those for particles on Keplerian orbits. Mukai \& Mann showed that, by taking into account the Doppler shift from the changing Sun-particle distance as well as the changing Earth-particle distance for particles on elliptical orbits and by allowing the force of radiation pressure to gravity $(\beta)$ to vary with heliocentric distance, the predicted Doppler shifts are in agreement with the observations.

\section{Dust bands and trails}

The maps of zodiacal emission obtained by the IRAS satellite in 1983 continue to be analyzed for structure in the zodiacal dust cloud. Sykes and Walker (Icarus 95, 180, 1992) have published a survey of cometary dust trails found in the IRAS data. The dust trails consist of large refractory particles ejected from comets at low velocities. Examination of the entire sky seen by IRAS has resulted in the detection of eight trails associated with short-period comets, as well as many faint trails having no known parent comet. Trails tended to be associated with objects having low perihelion distances that were observed near perihelion.

Detection of a solar ring of asteroidal particles in resonant lock with the Earth has been reported by Dermott et al. (presented at Div Plan Sci meeting, Oct. 1993, Bull AAS in press). This is manifested in the IRAS maps as an asymmetry in the ecliptic brightness of the zodiacal emission when viewed at a constant elongation angle of 90 degrees, with the trailing direction about $3 \%$ brighter than the leading direction.

Love and Brownlee (AJ 104, 2236, 1992) have examined the zodiacal dust bands in the 60 $\mu \mathrm{m}$ and $100 \mu \mathrm{m}$ IRAS bandpasses, where the contrast with the main zodiacal emission is larger than in the 12 and $25 \mu \mathrm{m}$ bandpasses. From the $60 \mu \mathrm{m}$ data, they estimate that the brightest dust bands contain approximately $0.2 \%-1.0 \%$ of the zodiacal dust. Analysis of the dynamical evolution of dust particles producing the bands is covered in Commission 22.

\section{Zodiacal light modeling}

The observed zodiacal light (or zodiacal emission) is an integral of the scattering (or emission) by all of the dust particles along the line of sight. From the observed properties of the zodiacal light, one wishes to derive the local values of fundamental quantities about the dust particles, such as their albedo, scattering function, degree of polarization, and temperature. Work on this topic has continued, using the "nodes of lesser uncertainty" method (Levasseur-Regourd, Renard, and Dumont, OED pp 131-138, 1991) to derive gradients of these quantities with heliocentric distance and with inclination from the dust symmetry plane. The local polarization degree decreases slightly with decreasing distance to the Sun between 1.5 and $0.3 \mathrm{AU}$ in the symmetry plane, with a steeper decrease close to the Sun; the polarization decreases with inclination from the symmetry plane (Renard et al. OED pp 199-202, 1991). There is clear evidence for more than one population of dust, each population having distinctive optical properties and orbital characteristics. This is not surprising, since both comets and asteroids are sources of interplanetary dust. Divine has modeled the interplanetary dust cloud as a complex of five populations with differing mass distributions and orbital characteristics in order to interpret space probe observations of interplanetary dust (Divine JGRP, in press, 1993).

The phase functions and polarization of interplanetary dust, cometary dust, and asteroids of different compositional classes, are being intercompared and also compared with circumstellar dust, in order to give clues to the origin of interplanetary dust. (Levasseur-Regourd, Renard, and 
Dumont, Adv Sp Res 11 No. 12, 175, 1991; Light scattering by irregular grains in comets and the interplanetary medium: Workshop on Polarization III, Levasseur-Regourd, Bastien, Hanner, eds, Aeronomie \& IAP Paris 1992)

Modeling of the F corona by I. Mann (Lindau) shows that white light observations yield information about optical properties of the particles if connected with accurate polarization measurements. The near-infrared spectral range gives a better description of the spatial distribution. More than half of the contribution to the brightness of the $F$ corona arises from dust near the Sun (r < 0.2 AU) (Mann A\&A 261, 329, 1992; Mann in MPB, pp 387-390, 1992).

\section{Optical Properties of Irregular Dust Particles}

To interpret the observations of dust particles in the solar system and the Galaxy, the optical properties of non-spherical particles need to be understood. The radiation pressure force on irregularly shaped interplanetary dust particles was examined by T. Mukai et al. (A\&A 262 315 1992) and their optical properties were studied in detail by Kozasa, Blum, and Mukai (A\&A 263423 1992) and Kozasa et al. (A\&A in press 1993). Orbital evolution of porous particles from comets and asteroids was investigated by Ishimoto et al. (in MPB, pp 65-68, 1993). Angular scattering measurements on aggregate particles, with and without absorbing mantles, using the Bochum microwave scattering laboratory, have been published by Zerull et al. (Applied Optics 32, 4088, 1993).

\section{GALACTIC AND EXTRAGALACTIC COMPONENTS}

\section{Ultraviolet background}

A wide field of view (8 degrees) far UV imager flown on the space shuttle in March 1992 obtained 22 images covering the bandpass $1400-1800 \AA$. These images have been analyzed to investigate Galactic and extragalactic diffuse radiation, as well as airglow and shuttle environment emission. (Lampton, Sasseen, Bowyer, Wu, GRL, 20, 539, 1993; Bowyer, Sasseen, Lampton, Wu, ApJ 415, in press, 1993). The Galactic FUV emissions show considerably more spatial structure than was previously thought, making the detection of the extragalactic emissions by their diffuse signals exceedingly difficult. Work in progress includes FUV properties of galaxies, FUV/IR correlations for diffuse emission from the ISM, Galactic stellar population studies for FUV stars, and FUV diffuse emission from dust.

The Far Ultraviolet Coronal Rocket established important new limits to diffuse spectral line emission in the $980-1080 \AA$ bandpass. The first useful limit to the emission measure of $O$ VI in the interstellar medium has been determined. The first directly measured limits to the filling factor of diffuse hot gas in the Galaxy have also been obtained (Edelstein and Bowyer, presented at COSPAR 29th Plenary Meeting, Adv Space Res, in press, 1993). The instrument was reflown in July 1992 as a secondary payload on the Delta launch vehicle as the Diffuse Ultraviolet Experiment (DUVE). The data obtained are now being analyzed and are expected to provide improved limits to FUV background emission lines, and to provide more stringent limits to diffuse FUV continuum radiation. 


\section{Optical Background}

K. Mattila (Helsinki) and Ch. Leinert (Heidelberg) have utilized the 2.2 and $1.23 \mathrm{~m}$ telescopes of the Calar Alto Observatory in parallel to continue measurements of the extragalactic background radiation with the dark cloud method (dark clouds Lynds 134 and 183, and the Draco cloud). Previous data of Mattila and Schnur (Bochum) from the ESO $1 \mathrm{~m}$ and 50 $\mathrm{cm}$ telescopes on Lynds 1642 have been further analyzed (Mattila, Leinert, Schnur, in Early Observable Universe from Diffuse Backgrounds. Proc. XIth Moriond Astrophysics Meeting, B. Rocca-Volmerange and J.-M. Deharveng, eds., Editions Frontieres, pp. 133-148, 1991).

In addition, the observations provide new information on the scattered light in dark nebulae and cirrus clouds for eight intermediate bands between 3500 and $8200 \AA$. Another byproduct is the determination of the sky brightness at these observatories and its variations during individual nights and from year to year.

\section{Infrared Background}

The COBE Science Working Group and their colleagues have been pursuing infrared background studies using data from the COBE Diffuse Infrared Background Experiment (DIRBE) (1.2 to $240 \mu \mathrm{m}$ wavelength) and the COBE Far Infrared Absolute Spectrophotometer (FIRAS) (0.01 to $1 \mathrm{~cm}$ wavelength). These instruments surveyed the full sky from November 1989 to September 1990, at which time the liquid helium coolant was exhausted. Since that time, the DIRBE has continued to collect data at $1.2,2.2,3.5$, and $4.9 \mu \mathrm{m}$ with a planned completion of the mission in December 1993. Initial COBE data sets were released to the scientific community in July 1993. The FIRAS data show that the spectrum of the cosmic microwave background (CMB) radiation does not deviate from a Planckian by more than $0.03 \%$ of the peak CMB brightness over the interval 0.05 to $0.5 \mathrm{~cm}$ wavelength (Mather et al., to be published in ApJLett 10 Jan 1994). This also implies that any contributions from galaxy formation to the cosmic infrared background (CIB) in this spectral range can not significantly exceed this level. The FIRAS also provided a survey of Galactic diffuse continuum and line emission. These data included the first detection of the bright $205 \mu \mathrm{m}$ [NII] line, as well as the most luminous cooling line of the interstellar medium, [CII] at $158 \mu \mathrm{m}$, an additional [NII] line, two [CI] lines, and 5 lines of CO (Wright et al., ApJ 381, 200, 1991).

DIRBE data have provided full sky absolute brightness maps in ten photometric bands from 1.2 to $240 \mu \mathrm{m}$, and linear polarization maps at 1.2, 2.2, and $3.5 \mu \mathrm{m}$. These measurements have high redundancy, providing detailed monitoring of the variation in sky brightness in every direction as the Earth moves within the interplanetary dust cloud. A preliminary measurement of the sky brightness in a relatively dark direction, the South Ecliptic Pole, which is a conservative upper limit on the CIB, was reported (Hauser et al., "After the First Three Minutes", AIP Conference Proc. 222, S. S. Holt, C. L. Bennett, \& V. Trimble (eds.), New York, p. 161, 1991). Reports on studies of diffuse galactic emission, including the shape of the stellar bulge, the shape of the stellar and interstellar components of the disk, stellar colors and extinction, physical conditions in the interstellar medium, and early polarization results, have also been presented ("Back to the Galaxy", AIP Conference Proc. 278, S. S. Holt \& F. Verter (eds.), New York, 1993). Present efforts focus on distinguishing foreground contributions from the solar system and Galaxy from the CIB. 